\title{
The Connection Between Radiation Pressure and Dispersion in Dielectric Waveguides
}

\author{
Peter T. Rakich ${ }^{1, *}$, Paul Davids ${ }^{1}$, and Zheng Wang ${ }^{2}$ \\ ${ }^{1}$ Sandia National Laboratories, PO Box 5800 Albquerque, NM 87185-1082 \\ ${ }^{2}$ Massachusetts Institute of Technology, 77 Massachusetts Ave., Cambridge, MA 02139 \\ *Corresponding author: rakich@alum.mit.edu
}

Compiled April 3, 2010

Through use of the response theory of optical forces, we show that radiation pressure generated within step index waveguides can be computed exactly and simply from the waveguide dispersion. In the absence of electrostrictive effects, we show that optical forces are only manifest on the boundaries of a dielectric waveguide. As a consequence, the principle of virtual work can be used to express the total optical force (or mean radiation pressure) acting on the boundaries of the waveguide in terms of the modal effective index. Through use of this relationship, exact agreement is found between the optical forces computed using waveguide dispersion and with the Maxwell stress tensor.

OCIS codes: $000.0000,999.9999$.

Recent studies of radiation pressure have revealed that large forces can be generated in nanometer-scale waveguides and cavities as a consequence of the high confinement and tremendous field enhancements generated within such systems [1-6]. In many cases, such optical forces can significantly impact the mechanics of bodies at micro- and nano- scales, yielding useful mechanical transduciton and actuation through use of micro- to milli-watt power levels [1-6].

In recent work, we have shown that radiation pressure is highly tailorable, and scales to large values in the context of high index-contrast optical waveguides, showing great promise in for use in novel optomechanical systems. In this paper, we use of the response theory of optical forces to demonstrate that radiation pressure generated within step index waveguides can be computed exactly and simply from the waveguide dispersion.

We begin by examining the spatial distribution of the optical forces generated in an idealized rectangular silicon waveguide of the type seen in Fig. 1. We assume that the elasto-optic coefficients are zero. As a consequence, all optically generated forces in the system can be attributed to radiation pressure [3]. The force distribution within the dielectric waveguide can be computed using the following form of the Maxwell stress tensor

$$
T_{i j}=\epsilon_{o} \epsilon\left[E_{i} E_{j}-\frac{1}{2} \delta_{i j}|E|^{2}\right]+\mu_{o} \mu\left[H_{i} H_{j}-\frac{1}{2} \delta_{i j}|H|^{2}\right] .
$$

Here, $E_{k}\left(H_{k}\right)$ is the $k^{t h}$ electric (magnetization) field component, $\epsilon_{o}\left(\mu_{o}\right)$ is the electric permittivity (magnetic permeability) of free space, and $\epsilon(\mu)$ is the relative electric permittivity (magnetic permeability). The force density (force per unit volume) generated from radiation pressure and gradient forces are computed from $T_{i j}$ as $\mathscr{F}_{j}^{r p}=\partial_{i} T_{i j}$.

Example force distributions generated by the TE-like mode within a rectangular silicon waveguide can be seen in Fig. 1. Figures. 1 (a)-(d) show the waveguide crossection and computed $E_{x}, E_{y}$, and $E_{z}$ electric field distributions corresponding to a silicon waveguide of width,
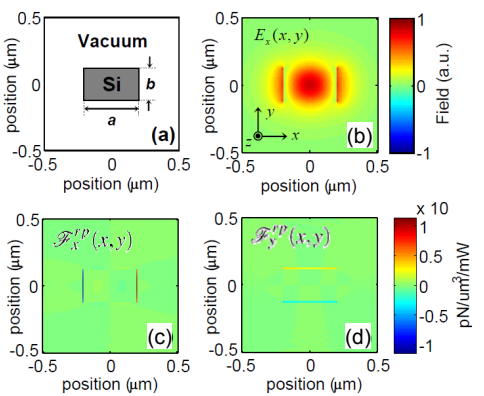

Fig. 1. (a) Schematic showing dimensions of silicon waveguide. (b) (c) and (d) show computed $E_{x}, E_{y}$, and $E_{z}$ components of TE waveguide mode. (c) and (d) show the time averaged $\mathscr{F}_{x}$ and $\mathscr{F}_{y}$ force densities.

$a=400 \mathrm{~nm}$, height, $b=250 \mathrm{~nm}$, and optical wavelength, $\lambda=1550 \mathrm{~nm}$. Figures $1(\mathrm{~g})$ and $(\mathrm{h})$ show the computed intensity maps of $\mathscr{F}_{y}^{r p}$ and $\mathscr{F}_{x}^{r p}$ components of the power normalized force density (force per unit volume) respectively, revealing that optical forces within the system (i.e., radiation pressure) appear to act only the boundaries of the waveguide system.

Moreover, one can prove that that optical forces vanish within the volume of the dielectric waveguide. Proof follows from the fact that the force density within dielectric media can be expressed as

$$
\mathscr{F}_{j}^{r p}=\partial_{i} T_{i j}=\frac{1}{2} \epsilon_{o}|E|^{2} \partial_{j} \epsilon(x, y) .
$$

Since the gradient of $\epsilon(x, y)$ vanishes for all positions excluding the boundary of the waveguide, one can see that no forces exist within the guide. This fact points to a simple means of computing radiation pressure in optical waveguides through virtual work.

Next, we develop a perturbative treatment of optical forces, leading to a simple relationship between the spatially averaged radiation pressure acting on the waveguide boundary and the waveguide effective index. A relationship of this form is useful since the radiation pressure 


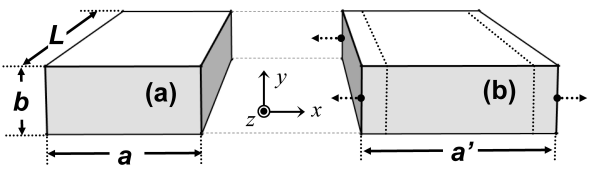

Fig. 2. (a) Rectangular waveguide segment of length, $L$, width, $a$, and height, $b$. (b) The right schematic shows the same waveguide after laterally strained by an and amount $\delta a$ to a new dimension of $a^{\prime}=a+\delta a$. Through this deformation, the waveguide height $(b)$ and length $(L)$ are held fixed.

acting on the various boundaries of the waveguide system can vary dramatically with waveguide dimension [3].

Through perturbative treatment, we consider the optical forces produced in silicon waveguides of the type seen in Fig. 2. Throughout, we assume that a guided optical mode, carrying an incident power, $P$, travels through TE waveguide mode. Using the principle of virtual work, we consider a virtual displacement of the waveguide boundaries, $\delta a$, and the change in total energy (or virtual work), $\delta U$, associated with this displacement. Through a virtual displacement, of the type illustrated in Fig. 2, the waveguide height $(b)$ and length $(L)$ are held fixed, while the waveguide width $(a)$ is varied. In general, the displacement-induced change in energy, $\delta U$, will be of the form $\delta U=\delta U_{M e c h}+\delta U_{E M}$, where $\delta U_{M e c h}$ is the mechanical work performed on the system through elastic deformation of the waveguide material in the absence of an electromagnetic field, and $\delta U_{E M}$ is the change in electromagnetic energy resulting from the waveguide deformation. In this case, the optical force total optical force acting to deform the waveguide in the $\mathrm{x}$-direction is simply

$$
F_{x}^{o p t}=-\left(\frac{\delta U_{E M}}{\delta a}\right)_{b} .
$$

To evaluate the radiation pressure induced force component using Eq. 2, we seek a simple means of evaluating $\delta U_{E M}$. Through use of th response theory of optical forces (RTOF), described in Ref. [2], one can show that the spatially averaged radiation pressure, $\bar{P}_{x}^{\text {opt }}$, acting on the lateral boundaries of the waveguide is

$$
\bar{P}_{x}^{o p t}=\frac{F_{x}^{o p t}}{P_{i} \cdot L \cdot b}=\frac{1}{c \cdot b}\left(\frac{d n_{e f f}(\omega, a, b)}{d a}\right)_{b}
$$

Above, $c$ is the speed of light in vacuum and $n_{e f f}$ is the effective index of the waveguide mode. An analagous relationship can be derived for the spatially averaged radiation pressure acting on the top and bottom boundaries. $\bar{P}_{y}^{\text {opt }}$. One can clearly see from Eqn. 3 that the spatially averaged radiation pressure can be simply computed from the variation in waveguide dispersion with waveguide dimension. This is powerful for the purposes of designing new optomechanical systems, since it relates the force to a simple scalar quantity, $n_{e f f}$. The derivative $d n_{e f f} / d a$ can be evaluated through perturbative methods, or numerically to compute the radiation pressure.

Through use of a full-vectorial mode solver, a comparison of this expression was made with that of the Maxwell
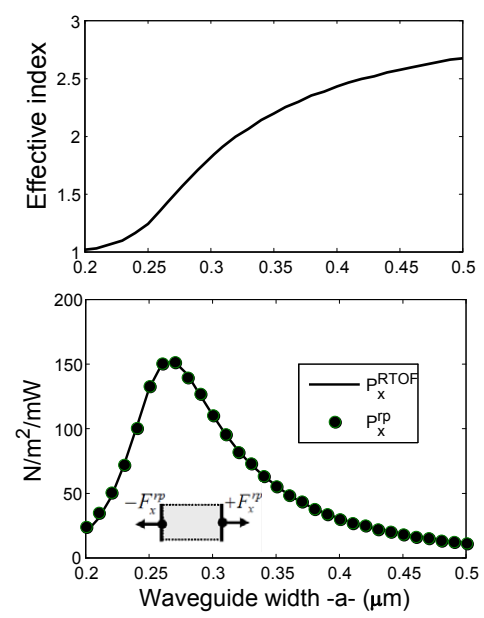

Fig. 3. (a) Effective index of rectangular waveguide as the waveguide width, $a$, is varied. (b) Shows computed spatially averaged radiation pressure using the Maxwell stress (circles) and RTOF (line).

stress tensor using a rectangular silicon waveguide over a range of waveguide dimensions. Figure 3 shows the results of this comparison. We find that the two methods agree within 0.1 percent. Furthermore, through use of Eqn. 1, one can show that Eqn. 3 is exactly equivalent to the forces computed using the Maxwell stress tensor.

In conclusion, through use of the response theory of optical forces, we have shown that radiation pressure generated in step index waveguides can be computed exactly and simply from the waveguide dispersion. We will also show that electrostrictive forces generated within the waveguide can also be treated in a similar manner. These results are powerful for the purposes of designing optomechanical systems, since relation of forces to a simple scalar quantity, such as $n_{e f f}$, greatly simplifies the optimization of an optomechanical behavior.

Sandia laboratory is operated by Sandia Corporation, a Lockheed Martin Company, for the US Department of Energy's NNSA under contract DE-AC04-94AL85000. This work was supported by the Director of Defense Research and Engineering under Air Force contract FA8721-05-C-0002 and through a Seedling managed by Dr. Mike Haney of DARPA MTO.

\section{References}

1. P. Rakich, M. Popovic, M. Soljacic, and E. Ippen, Nature Photonics, vol. 1, no. 11, pp. 658-669, 2007.

2. P. Rakich, M. Popovic, and Z. Wang, Optics Express, vol. 17, no. 20, pp. 18116-18135, 2009.

3. P. Rakich, P. Davids, and Z. Wang, "Tailoring Optical Forces in Waveguides Through Radiation Pressure and Electrostrictive Forces," In preparation: Optics Express.

4. M. Li, W. H. P. Pernice, C. Xiong, et al, Nature, vol. 456, pp. 480, Nov 272008.

5. M. Povinelli, M. Loncar, M. Ibanescu et al, Optics Letters, vol. 30, pp. 3042-3044, Nov 152005.

6. M. Eichenfield, C. P. Michael, R. Perahia et al, Nature Photonics, vol. 1, pp. 416-422, JUL 2007. 